\title{
The Impact of Preoperative Assessment and Planning on the Outcome of Benign Hysterectomy - a Systematic Review
}

\author{
Auswirkung von präoperativer Untersuchung und \\ Planung auf das Outcome nach benigner Hysterektomie - \\ ein systematischer Überblick
}

\section{(ㄷ) (1) (옹}

Authors

Lollo Makdessi Björkström ${ }^{1}$, Ninnie Borendal Wodlin², Lena Nilsson ${ }^{3}$, Preben Kjølhede ${ }^{2}$

\section{Affiliations}

1 Department of Obstetrics and Gynecology in Norrköping, and Department of Biomedical and Clinical Sciences, Linköping University, Linköping, Sweden

2 Department of Obstetrics and Gynecology in Linköping, and Department of Biomedical and Clinical Sciences, Linköping University, Linköping, Sweden

3 Department of Anesthesiology and Intensive Care in Linköping, and Department of Biomedical and Clinical Sciences, Linköping University, Linköping

Key words

benign hysterectomy, preoperative planning, ERAS

Schlüsselwörter

benigne Hysterektomie, präoperative Planung, ERAS

received 21.4.2020

accepted after revision

13.9. 2020

Bibliography

Geburtsh Frauenheilk 2021; 81: 200-213

DOI 10.1055/a-1263-0811

ISSN 0016-5751

(c) 2021. The Author(s).

This is an open access article published by Thieme under the terms of the Creative Commons Attribution-NonDerivative-NonCommercial-License, permitting copying and reproduction so long as the original work is given appropriate credit. Contents may not be used for commercial purposes, or adapted, remixed, transformed or built upon. (https://creativecommons.org/licenses/by-nc-nd/4.0/)

Georg Thieme Verlag KG, Rüdigerstraße 14,

70469 Stuttgart, Germany

Correspondence

Lollo Makdessi Björkström, MD

Department of Obstetrics and Gynecology

Vrinnevi Hospital, 60182 Norrköping, Sweden

Lollo.Makdessi.Bjorkstrom@regionostergotland.se

\section{ABSTRACT}

Knowledge concerning the impact of preoperative planning, patient information and patient factors on the outcome of benign hysterectomy is incomplete. This systematic review summarizes the current knowledge on the effect of preoperative planning and of preoperative patient factors on the outcome of benign hysterectomy. The PubMed/PubMed Central/MEDLINE, Scopus, Web of Science, TRIP Medical Database, Prospero and the Cochrane Library databases were searched. Inclusion criteria were prospective trials, hysterectomy for benign disease, systematic preoperative assessment, and article in English. Eighteen articles were included and categorized according to their main aims: use of a preoperative checklist, preoperative decision-making, preoperative information, and the effect on the outcome of surgery of factors that concerns patients preoperatively. Focused and well directed preoperative assessment and thoroughness in the preoperative decision-making was associated with positive postoperative outcomes. The use of a checklist reduced the overall rate of hysterectomy and increased the use of minimally invasive surgery. Women were often inadequately informed before hysterectomy about the possible side effects after surgery. Preoperative anxiety and preoperative pain were associated with postoperative pain and lower quality of life. The indication for surgery had an impact on the reported quality of life postoperatively. The extent of preoperative planning seemed to affect the outcome of surgery. Preoperative patient factors influenced the postoperative recovery. Prehabilitation measures need further development and should be integrated in the preoperative planning. Prospective studies are warranted to evaluate and improve the preoperative planning in a systematic setting before performing hysterectomy for benign disease. 


\section{ZUSAMMENFASSUNG}

Das Wissen um die Auswirkung von präoperativer Planung und Patientenaufklärung sowie von patientenbezogenen Faktoren auf das Outcome nach benigner Hysterektomie ist unvollständig. Diese systematische Übersichtsarbeit fasst den aktuellen Wissensstand über die Auswirkung von präoperativer Planung und präoperativen patientenbezogenen Faktoren auf das Outcome nach benigner Hysterektomie zusammen. Es wurde zunächst eine Suche in den Datenbanken PubMed/ PubMed Central/MEDLINE, Scopus, Web of Science, TRIP Medical Database, Prospero und the Cochrane Library durchgeführt. Die Einschlusskriterien waren: prospektive Studie, Hysterektomie bei benigner Erkrankung, systematische präoperative Untersuchung, Artikel in englischer Sprache. Insgesamt wurden 18 Artikel in die Studie aufgenommen und nach ihren wesentlichen Zielen kategorisiert, wie folgt: Verwendung einer präoperativen Checkliste, präoperative Entscheidungsfindung, präoperative Aufklärung, die Auswirkung von patientenbezogenen präoperativen Faktoren auf das Outcome nach dem erfolgten Eingriff. Zielgerichtete präoperative
Untersuchungen und eine gründliche und sorgfältige präoperative Entscheidungsfindung waren mit positiven postoperativen Ergebnissen assoziiert. Die Verwendung einer Checkliste verringerte die Gesamtrate an Hysterektomien und erhöhte die Anzahl minimalinvasiver Eingriffe. Frauen wurden vor der Hysterektomie oft nur unzureichend über die möglichen Nebenwirkungen des Eingriffs aufgeklärt. Präoperative Angst und präoperative Schmerzen waren mit postoperativen Schmerzen und geringer Lebensqualität verbunden. Die Indikation für den Eingriff hatte auch einen Einfluss auf die beschriebene Lebensqualität nach der Operation. Das Ausmaß an präoperativer Planung beeinflusste das Ergebnis nach der Hysterektomie. Präoperative patientenbezogene Faktoren wirkten sich auf die postoperative Heilung aus. Der Einsatz gezielter Aufbaumaßnahmen vor chirurgischen Eingriffen sollte ausgebaut und in die präoperative Planung integriert werden. Prospektive Studien sind gefragt, um die präoperative Planung vor Hysterektomien bei benigner Erkrankung systematisch auszuwerten und zu verbessern.

\section{Introduction}

Hysterectomy for benign gynecological disorders is the most common major gynecological surgical procedure [1] even though the rate of benign hysterectomy has shown a downward trend in Western countries during the recent decades. The lower rate is essentially caused by improved medical treatment of some of the most common indications for hysterectomy [2]. The most frequent benign indications for hysterectomy are fibroids, abnormal uterine bleeding (AUB), pelvic pain, cervical dysplasia and uterine prolapse [3-5].

The preoperative planning and decision-making for hysterectomy is extensive and comprises several important elements. A trusting patient-doctor relationship is essential but in addition, the patient's needs and requests, the indications for the surgery, alternative treatment options, and the expectations of what can be achieved by the treatment should be discussed thoroughly. The selection of the route of hysterectomy for benign causes is influenced by the patient's characteristics, the surgeon's experience, and the attitudes and preferences of the informed patient [5-8]. The risk for complications and undesirable outcome must be considered when deciding on surgery, especially in the case of benign conditions where the intention is to improve health-related quality of life (QoL) [9-12].

The complex processes of preoperative decision-making and medical consultation and planning are subjects of major importance as they may affect the outcome [13]. The "enhanced recovery after surgery" (ERAS), or "fast-track surgery" is a framework with the purpose of controlling perioperative pathophysiology, and subsequently enhancing the recovery. ERAS consists of multidisciplinary ingredients in which preoperative preparation and preoperative information to patients are elements. However, due to the multimodality concept of ERAS the contribution and specif- ic effects of the preoperative preparation and information on the overall result of ERAS are difficult to evaluate [8].

More recently, the concept of prehabilitation has evolved in order to further improve recovery after surgery [14] and has been proposed to be integrated in ERAS programs [15]. Prehabilitation is defined as the practice of enhancing a patient's functional capacity before surgery, with the aim of improving postoperative outcomes [14].

The preoperative consultation should include information about the recovery period, especially concerning the occurrence of postoperative symptoms and about possible long-term effects on chronic pain, sexual functioning and the QoL, since these factors are known to be of concern for women before hysterectomy. The patient-reported outcome and experience measures such as QoL, satisfaction and postoperative symptoms have attracted increasing public and medical interest. Knowledge of preoperative factors that might adversely influence the course and eventually the outcome of surgery is essential in order to improve the perioperative care and the postoperative recovery $[5,13]$. However, it would be of more interest to examine whether specific interventions in relation to the surgery are effective in preventing these long-term adverse effects.

There is no generally accepted uniformity in the preoperative procedures before benign hysterectomy in clinical practice, and the knowledge about the impact of preoperative planning and targeted patient information in a systematic setting on the outcome of benign hysterectomy is still incomplete.

In this descriptive systematic review, we aim to summarize the contemporary evidence on whether systematic preoperative planning for benign hysterectomy improves the postoperative outcome, with special emphasis on patient-reported measures and adverse outcomes, and use of prehabilitation. We also summarize the current knowledge regarding factors that concerns patients preoperatively of importance for the postoperative recov- 
ery since these should be targeted specifically in systematic preoperative planning, and specifically we evaluate the ability of preventive interventions to reduce the negative impact of these factors on recovery.

\section{Review}

A search for clinical studies that focused on systematic preoperative planning including the impact of preoperative characteristics, and that evaluated the effects on the outcome of surgery for benign hysterectomy was performed, as well as a computer-aided literature search in the National Library of Medicine (PubMed/ PubMed Central/MEDLINE), Scopus, Web of Science, TRIP Medical Database, Prospero and the Cochrane Library databases covering the period from January 1990 to June 2020. The following keywords (in alphabetic order) were used: algorithm, appointment, assessment, attitude, benign, counsel, counseling, enhanced recovery after surgery (ERAS), evaluation, fast track, flow chart, gynecological, gynecology, hysterectomy, intervention, investigation, life-style, management, patient-reported experience measure, patient-reported outcome measure, planning, prehabilitation, preoperative, preparation, procedures, protocol, quality of life, score, standardized, systematic, care, tool. AND/OR was used between the different search terms.

Studies were included if they met the following criteria:

1. prospective trials,

2. hysterectomy for benign disease,

3. systematic preoperative assessment, and

4. article in English.

Doublets, studies that included cancer surgery, retrospective studies, and studies with previously published results were excluded. In addition, studies that focused on perioperative preemptive interventions such as use of antibiotics, thromboprophylaxis or vaginal cleansing, or studies with multi-modal intervention where the preoperative assessment could not be solely evaluated were likewise excluded.

The reference lists in all identified relevant articles and reviews were searched for additional published studies concerning the topic of preoperative planning for benign hysterectomy.

The study was conducted according to the PRISMA guidelines [16]. The process of searching for and selecting articles from the search hits was based on the PICOS (Patient/population - Intervention - Comparative controls - Outcomes - Statistical analyses) framework, described by the Centre for Reviews and Dissemination [17].

Briefly, the search hits were initially read by titles. Articles were excluded if it appeared that the title was irrelevant to the scope of this systematic review. The abstracts of the remaining articles were read. Those that were not within the scope of this review were excluded. The remaining articles were read in full text for a detailed quality assessment of whether they complied with the area of this systematic review.

The total number of search hits was 4684 , of which 4453 were excluded after reading the article titles. The abstracts of 231 articles were scrutinized, leaving 62 articles for a full text review.
Eventually, 18 articles remained to be included in the systematic review.

None of the studies had the primary aim of comparing the effect of systematic preoperative planning on the outcome of surgery for benign hysterectomy. The articles included described preoperative assessment and prospective measures of importance for the postoperative outcomes. Due to the substantial differences in the outcome measures, it was not meaningful to enter the results into a statistic meta-analysis model. Consequently, the results are presented as a descriptive systematic review article.

The articles were categorized according to the subject of their main aim, i.e. as about one of the following:

1. use of a preoperative checklist,

2. preoperative decision-making,

3. preoperative information,

4. the effect of the preoperative patient-concerned factors on the outcome of surgery and effect of preventive interventions on these, and

5. prehabilitation.

Three of the trials were interventional, one was cross-sectional, and the remaining 14 were longitudinal cohort studies. > Table 1 summarizes the current knowledge from the five published studies concerning a systematic preoperative assessment before benign hysterectomy. - Table 2 summarizes the 13 studies that studied the effect of the preoperative patient factors, patients' goals, mental health, pain, and sexual function and QoL on the outcome of surgery.

\section{Use of a preoperative checklist}

Two interventional studies compared the effect of the use of a preoperative checklist $[18,19]$; one focused on the rate of hysterectomy and found a lower rate of hysterectomy when using a checklist [19]. The other considered the route of hysterectomy and found an increased rate of vaginal hysterectomy when using a checklist [18]. Kovac et al. examined resident clinic doctors' use of the guidelines of the Society of Pelvic Reconstructive Surgeons as a checklist for a preoperative determination of the route of hysterectomy [18]. Their study included 407 women, of which 88 had suspected conditions such as endometriosis and/or adhesions that could complicate the vaginal approach. For the entire group, vaginal hysterectomy was completed successfully in $91.8 \%$ of the subjects, resulting in a change in the ratio of abdominal-to-vaginal hysterectomy from $3: 1$ to $1: 11$, during a period of five years. The other study by Hullfish et al. investigated the effect of the use of a checklist on the final histology and rates of hysterectomy for benign conditions [19]. The study included fibroids, chronic pelvic pain and bleeding as indications for hysterectomy and showed a significantly lower rate of hysterectomy and a lower rate of nonconfirmable pathology when using a checklist during a six-month period.

Thus, the use of a checklist may have impact on the rate of surgical intervention [19] and the choice of surgical approach may also be affected by use of a checklist [18]. 


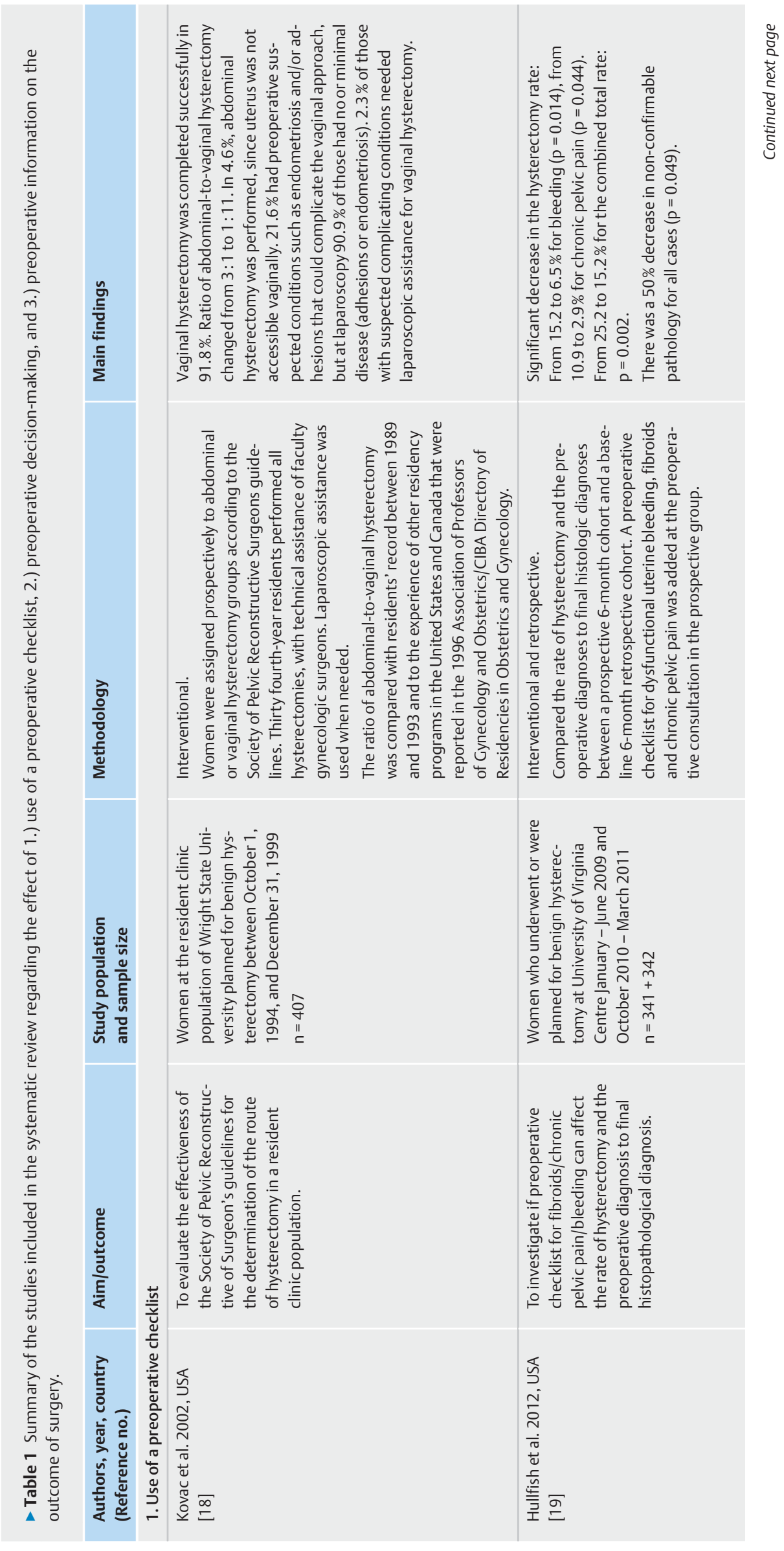




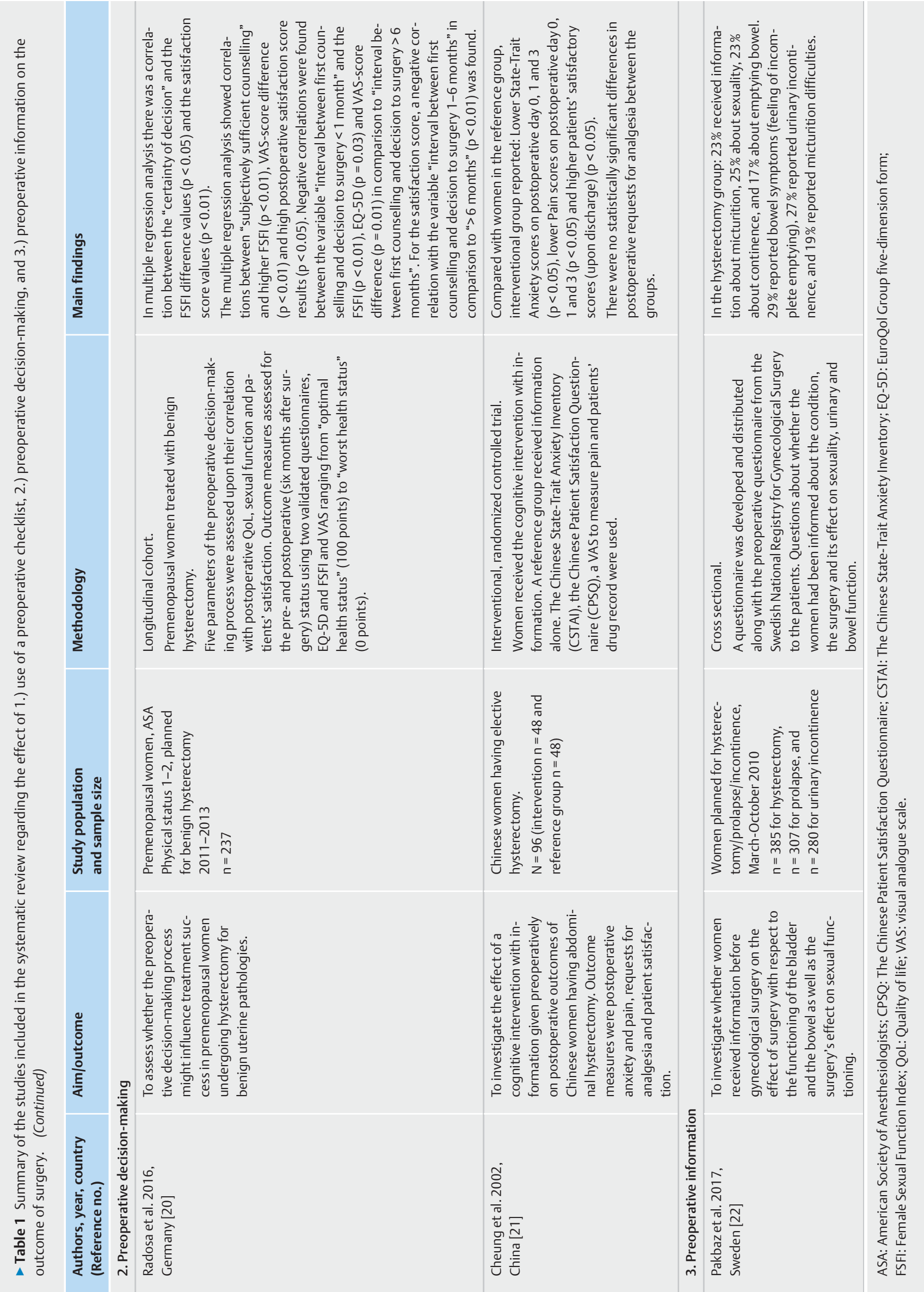




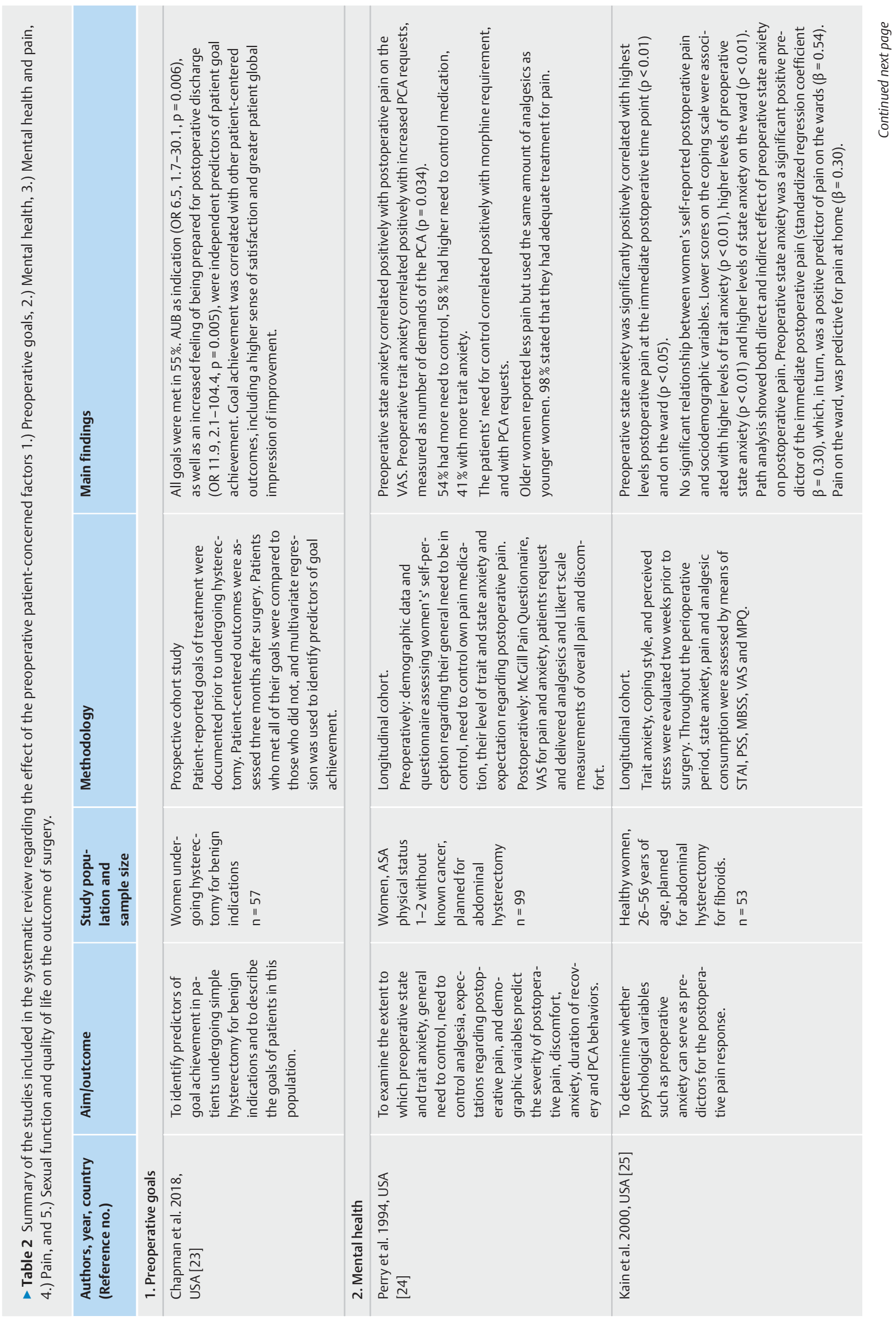




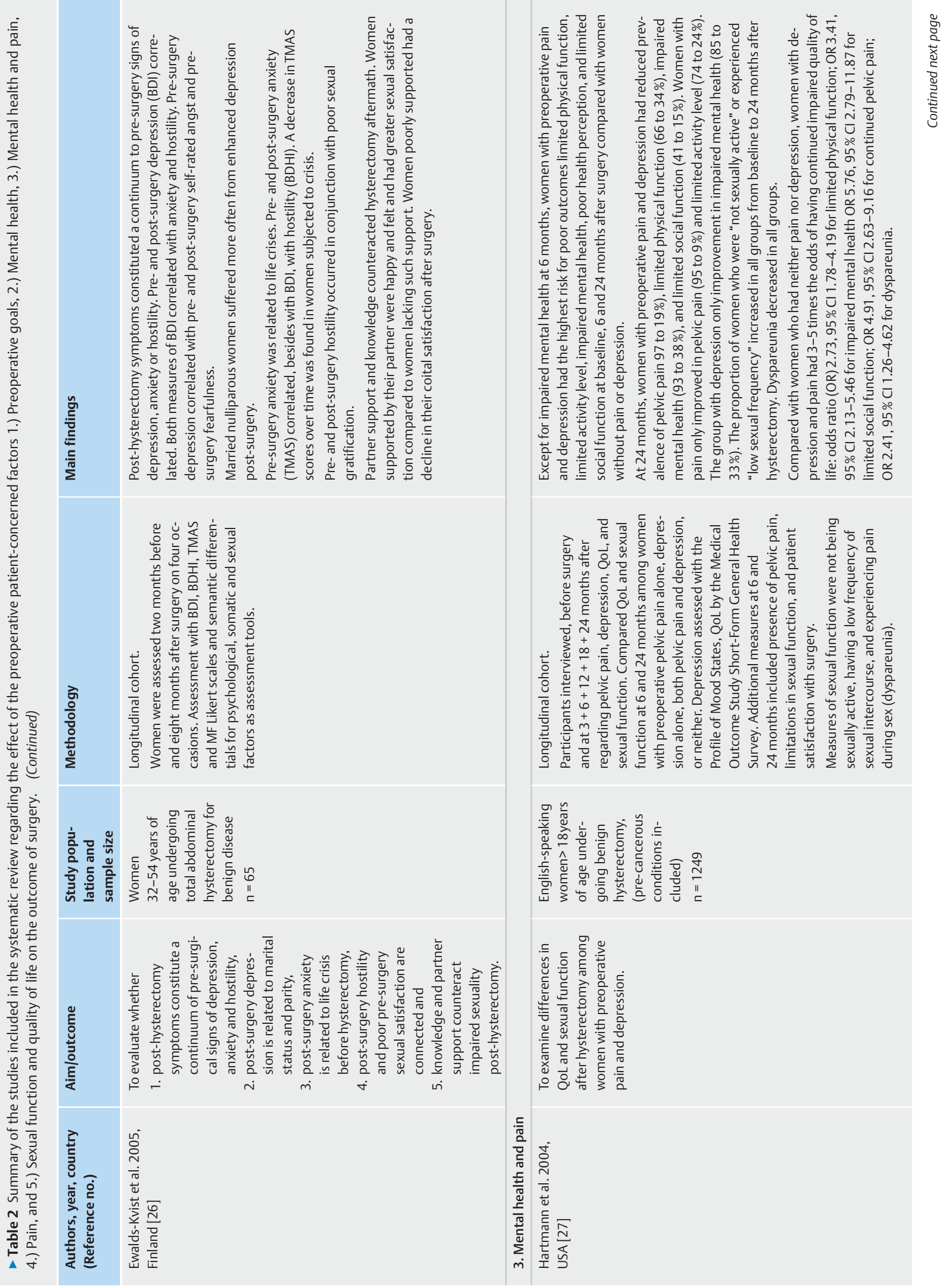




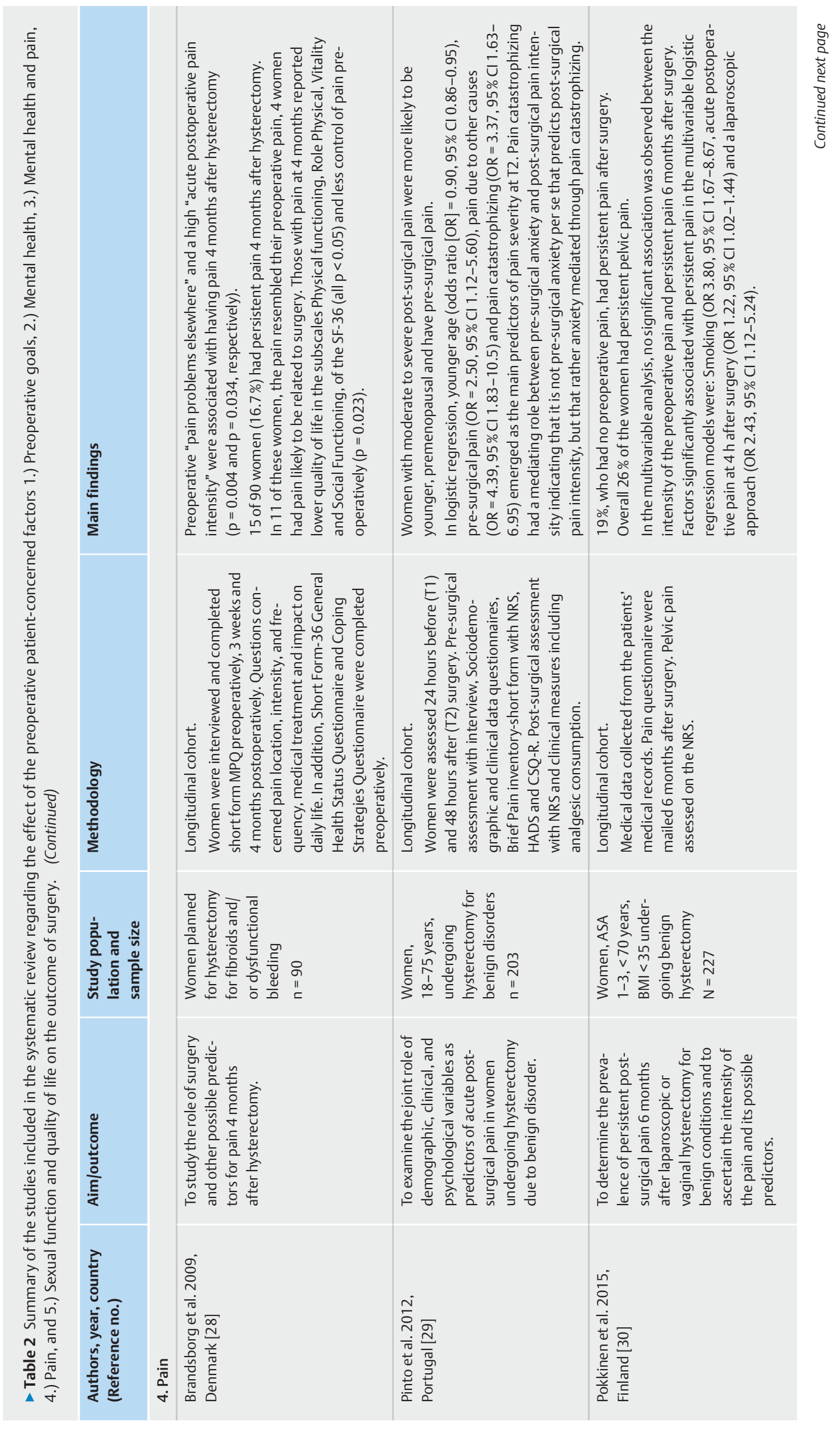




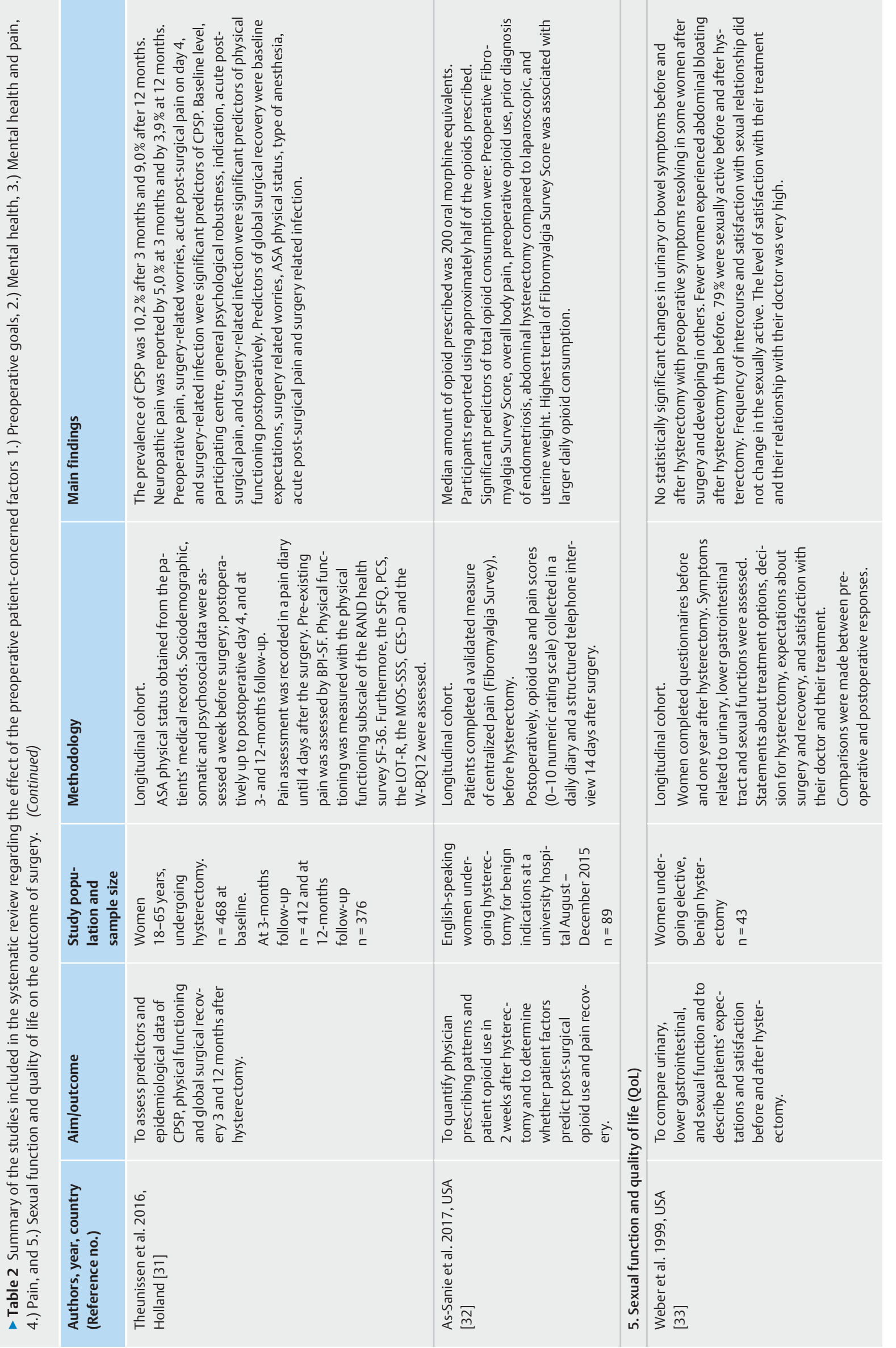




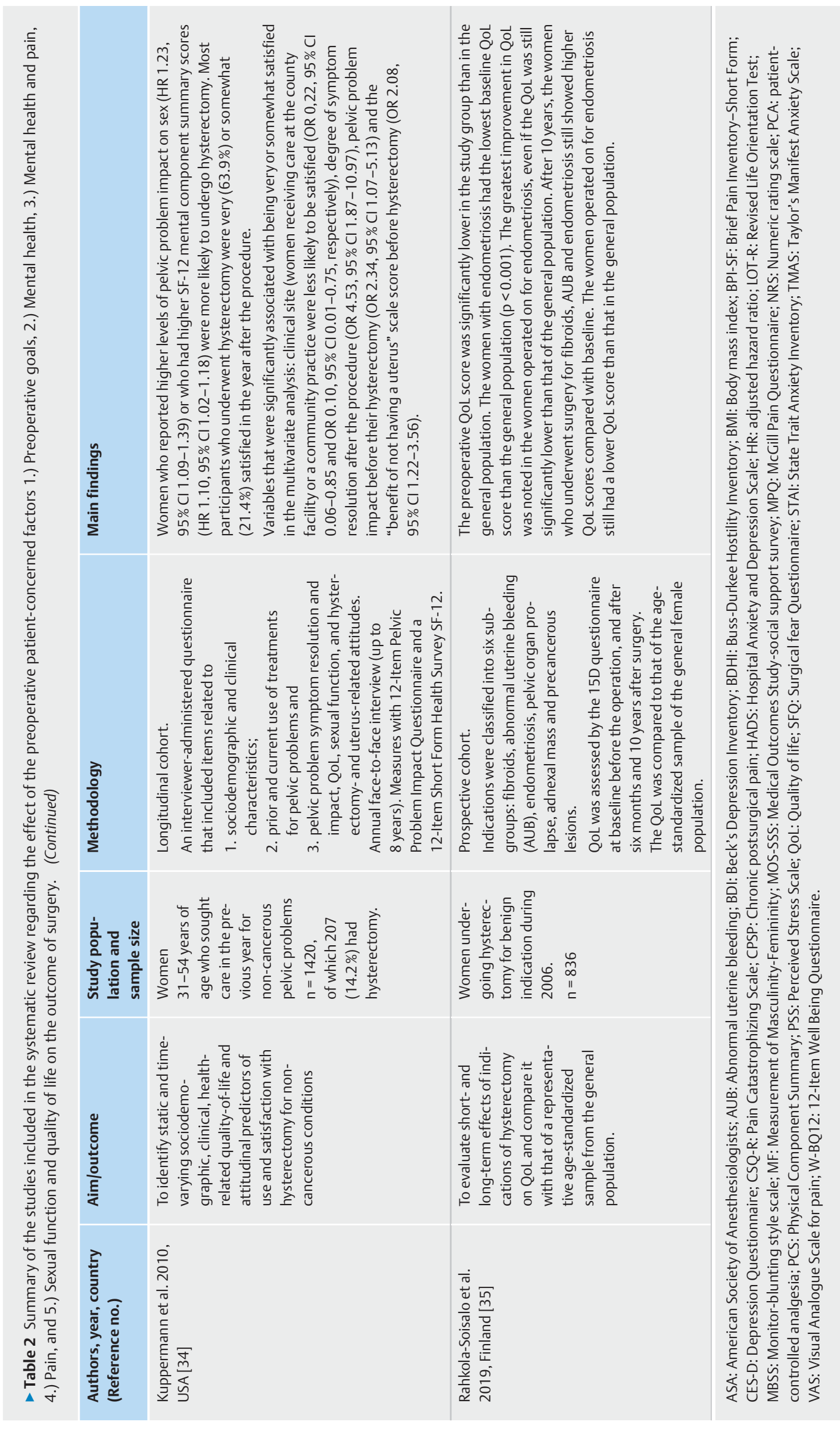




\section{Preoperative decision-making}

Only one study that dealt with the preoperative decision-making on hysterectomy for benign disease was found. The authors assessed whether the preoperative decision-making process in women undergoing hysterectomy for benign uterine pathologies influenced the success of treatment, measured by patient-reported measurements focusing on the sexual function, QoL and satisfaction [20]. Women who were convinced to opt for surgery had a higher sexual function index and higher satisfaction scores postoperatively. There was a positive correlation between "subjectively sufficient counselling" and higher sexual function index and high postoperative satisfaction scores.

Thus, it seemed that systematic patient involvement in the decision process may affect the patient-reported measures [20].

\section{Preoperative information}

A Chinese trial studied the effect of distraction and reappraisal coping techniques together with an information booklet, given preoperatively, on postoperative outcomes compared with information alone on women undergoing hysterectomy [21]. The booklet contained information about general preoperative care, simple anatomy and physiology of the uterus, the hysterectomy procedure, common advice after surgery and misconceptions about postoperative outcomes. The intervention group received the same booklet as the reference group with the addition of written instructions on how to apply coping techniques. The women who received the intervention reported lower postoperative anxiety scores, lower pain scores and higher levels of satisfaction than the women in the reference group.

A Swedish study investigated whether women received information before benign hysterectomy about the effect on sexuality and the function of the bladder and bowel [22]. Only one out of four women undergoing hysterectomy claimed to have received information about the effect of the surgery on sexual life and bladder function, and $17 \%$ received information about bowel function.

Preoperative information concerning post-surgical effects on sexuality, bowel, and bladder function is insufficient, and coping strategies might help women to handle this type of problem [21, 22].

\section{The effect on the outcome of surgery of factors that concern patients preoperatively}

The preoperative patient-concerned factors that could affect the outcome of surgery examined in the included studies were divided into five main categories: preoperative goal, mental health, pain, sexual functioning and QoL. Some of the studies examined more than one of these preoperative factors and their association with the outcome of hysterectomy.

\section{Preoperative goals}

Only one study was identified that dealt with predictors of goal achievement in patients undergoing benign hysterectomy, and described the general profile of patient goals in order to gain better insight into the patients' personal motivations and expectations surrounding hysterectomy [23]. Prior to surgery the patients were asked to list one to three goals they hoped to achieve through hysterectomy. After surgery the patients were asked to assess the achievement of each of their goals. All goals were met in $55 \%$ (26 of 47 patients) whereas $45 \%$ did not meet any goal. The study also revealed that the patients undergoing hysterectomy for AUB as the primary indication, those who felt more prepared for postoperative discharge and those with shorter hospitalization were more likely to reach complete goal achievement. The goals were categorized dichotomously as symptomatic (bleeding, pain or discomfort, bowel symptoms, urinary symptoms) or functional (related to overall health and well-being, quality of life, safety and recovery concerns, and removal of unwanted organs). The patients were more concerned with symptomatic goals than functional goals.

Thus, patients undergoing hysterectomy may have specific goals with the surgery, which should be addressed in the preoperative counselling [23] in order to ensure that the goals are realistic and attainable.

\section{Mental health}

All three studies that investigated the associations between preoperative mental health and postoperative outcome found that the preoperative mental health correlated proportionally with the outcome after surgery. None of the studies was interventional.

Demographic variables, preoperative anxiety, self-rated general need to control, self-rated need to control one's own pain medication and expectations regarding postoperative pain were evaluated in one study for the prediction of the severity of postoperative pain, discomfort, anxiety, duration of recovery and patientcontrolled analgesia (PCA) behaviors [24]. Older women reported less pain, even though they used the same amount of analgesic medication as younger women. Preoperative trait anxiety correlated with increased analgesic requests measured as button presses on the PCA, but not with postoperative pain. The preoperative signs of anxiety correlated with postoperative pain and with a shorter hospital stay. The women's need to be in control was associated positively with the morphine requirement, and with analgesic requests made with the PCA. The authors concluded that psychological factors influenced postoperative recovery and pain control in women receiving PCA therapy after abdominal hysterectomy, and that individualized treatment may lead to improved postoperative outcomes.

However, another study found both direct and indirect effects of the preoperative signs of anxiety on postoperative pain up to one week postoperatively [25]. The preoperative state of anxiety was a significant positive predictor of the immediate postoperative pain that, in turn, was a positive predictor of pain experienced later in the ward. Perceived pain in the ward was predictive for continued experienced pain at home.

A Finnish study evaluated the impact of psychological and sexual factors on the outcome of hysterectomy up to eight months after surgery [26]. The study showed that post-hysterectomy symptoms constituted a continuum to pre-surgery signs of depression, anxiety or hostility. Pre- and post-surgery depression were both directly correlated. Married nulliparous women suffered more from enhanced depression post-surgery. Pre- and post-surgery hostility occurred in conjunction with poor sexual 
gratification. Partner support and knowledge counteracted the hysterectomy aftermath. Women who were supported by their partner were happy and had greater sexual satisfaction compared with women lacking such support. Women who were poorly supported had a decline in their coital satisfaction after surgery.

\section{Mental health and pain}

In a study from the USA, Hartmann et al. examined differences in QoL and sexual function among women undergoing hysterectomy with preoperative pelvic pain alone, depression alone, both pelvic pain and depression, or neither [27]. Their results showed that 24 months postoperatively, the women with both pain and depression had a reduced prevalence of pelvic pain, limited physical function, impaired mental health, and limited social function. The women with only pelvic pain improved concerning pelvic pain and activity level. The group with only depression improved in mental health. Dyspareunia decreased in all groups. Compared with women who had neither pain nor depression, the women with depression and pain had three to five times the risk of continued impaired quality of life.

\section{Pain}

Five studies showed an association between preoperative pain and postoperative recovery. Brandsborg et al. found that preoperative "pain problems elsewhere" and a high "acute postoperative pain intensity" were associated with persistent pain four months after hysterectomy [28]. Women with lower QoL and less control of pain preoperatively reported more frequent pain at four months. Pinto et al. examined the joint role of demographic, clinical, and psychological variables [29]. Younger age, pre-surgical pain, pain due to other causes, and pain catastrophizing behavior were the main predictors of acute post-surgical pain severity after hysterectomy due to benign disorders. The pre-surgical anxiety also predicted the pain intensity. Pain catastrophizing behavior seemed to be a mediator between pre-surgical anxiety and experienced post-surgical pain intensity.

Pokkinen et al. found that one of four women had chronic post-surgical pain (CPSP) six months after laparoscopic or vaginal hysterectomy for benign causes [30]. Furthermore, that trial aimed to examine the intensity of pain and its possible predictors. Most of the women rated their average pain as mild. In the multivariable analysis, no significant association was observed between the intensity of the preoperative pain and persistent pain six months after surgery. Smoking, acute postoperative pain at four hours after surgery and a laparoscopic approach were significantly associated with persistent pain in the multivariable analysis.

Theunissen et al. assessed predictors and epidemiological data of CPSP, physical functioning and global surgical recovery three and 12 months after hysterectomy and found that preoperative pain, surgery-related worries, acute post-surgical pain, and surgery-related infection were significant predictors of CPSP [31]. The baseline physical functioning level, participating center, general psychological robustness, indication for surgery, acute postsurgical pain, and surgery-related infections were significant predictors of physical functioning postoperatively.

A study from the USA quantified physicians' prescribing patterns and women's opioid use during the two weeks after hyster- ectomy and determined whether patient factors predicted postsurgical opioid use and pain recovery [32]. The median amount of opioid prescribed was 200 morphine equivalents orally, and the women reported using approximately half of the opioids prescribed. The total opioid consumption correlated to overall body pain, preoperative opioid use, prior endometriosis, abdominal technique and uterine weight. A personalized approach to prescribing opioids for postoperative pain could be considered.

\section{Sexual function and QoL}

Two studies had the main aim of assessing the preoperative sexual function and/or the QoL before and after hysterectomy. Hysterectomy had a positive effect on the QoL. Weber et al. compared urinary, lower gastrointestinal, and sexual function before and after hysterectomy and described women's expectations and satisfaction [33]. They did not find statistically significant changes in urinary or bowel symptoms, and the sexual function did not change in the sexually active women. The level of satisfaction with the treatment was very high.

In a large prospective longitudinal cohort study of 1420 women from the USA, Kuppermann et al. identified static and timevarying sociodemographic, clinical, QoL and attitudinal predictors of use and satisfaction with hysterectomy for non-cancerous conditions, up to eight years after surgery [34]. Most participants who underwent hysterectomy were satisfied in the year after the procedure. The authors concluded that there were numerous factors beyond clinical symptoms that predicted hysterectomy use and satisfaction, and that QoL, sexual function, and attitudes should be discussed with women prior to surgery.

The recently published FINHYST study by Rahkola et al. demonstrated that the indication of benign hysterectomy had a significant impact on the recovery of QoL even in the long-term, up to 10 years after the operation [35]. The women in all categories of indications of hysterectomy revealed significantly lower scoring in QoL preoperatively than a group of age-standardized women from the general population. Women with endometriosis as an indication had the lowest QoL score at baseline. Postoperatively, this group presented the greatest improvement in QoL score but the QoL score was still lower than that of the standardized general population. After 10 years, the women in all categories of indications except endometriosis had QoL scores that were higher than that of the standardized general population. This indicated that women operated with hysterectomy on the indication of endometriosis did not achieve a level of QoL comparable to that of healthy women.

Hysterectomy has a positive effect on QoL [29,33-35]. The postoperative QoL was associated with the indication for surgery even after 10 years of follow-up [35]. Preoperative mental health, especially anxiety, pain catastrophizing, and preoperative pain were associated with postoperative pain and lower QoL [25-28].

Knowledge of the effect of preoperative patient factors on the outcome of surgery is essential for the preoperative assessment. Pre-surgical factors can be assessed to help identify those who are most likely to benefit from the procedure and those who are at risk of experiencing a less favorable outcome. The preoperative factors of importance for postoperative recovery in our review were mental health, pain, sexual function and QoL. However, in 
spite of the knowledge of risk factors for adverse outcome after hysterectomy very few studies have been designed to investigate the effect of preventive interventions.

The risk of an undesirable outcome must be carefully considered when deciding on surgery, especially in the case of surgery for benign conditions. In contrast to many other areas of benign hysterectomy, for example the indication and mode of surgery, surgical technique, intra- and postoperative complications, the field of systematic preoperative planning is largely unexplored. The results from the presented studies strongly indicated an association between the preoperative procedures and the postoperative outcomes.

\section{Prehabilitation}

Prehabilitation programs may include any or all of four main components:

1. medical optimization

2. physical exercise,

3. nutritional support, and

4. psychological support before surgery to improve the overall conditioning of the patient [14].

The medical optimization usually covers preoperative smoking cessation, reduction in alcohol intake, weight reduction (provided the patient is overweight or obese), management of anemia and optimization of pharmaceutical therapy of medical conditions such as diabetes mellitus, hypertension, heart disease and chronic pulmonary disease. The physical exercise is primarily aimed at increasing the capacity of the functional airways and heart capacity, but also the general fitness. At the same time it might facilitate weight reduction. Nutritional support includes carbohydrate loading preoperatively as well as preoperative nutrition therapy of malnourished or starving individuals. Psychological support has a dual purpose, to reduce psychological distress and anxiety associated with diagnosis and surgery, and to maximize patients' motivation and empower them to comply with the exercise and nutritional aspects of the program.

None of the studies in this review included prehabilitation in the preoperative assessment. A review showed that prehabilitation can reduce overall and pulmonary morbidity following major abdominal surgery, but the target population for prehabilitation programs remains to be established [36]. In 2018, Ebner et al. presented a review of gynecological studies dealing with optimal surgical self-preparation - prehabilitation - before hysterectomy [37]. They found a limited number of studies and these had low numbers of patients. Although there seemed to be a benefit to earlier discharge after prehabilitation, they concluded that further studies in gynecology are needed to determine the advantages for the patients.

Several studies have demonstrated that preoperative change of lifestyle factors such as physical activity, tobacco smoking and alcohol can improve the outcome of surgery [38-40]. The number of studies investigating the effect of change of lifestyle within gynecological surgery is very limited $[37,41]$.

\section{Strength and limitations}

The strength of this review is the systematic and thorough research of the literature using the PRISMA guidelines. A limitation is the small number of studies that met the review's inclusion and exclusion criteria. Moreover, only one study was randomized, and the others were cross-sectional or longitudinal cohort studies. This implies a high risk of bias in these studies. Thus, the results of the review should be interpreted with great caution.

\section{Conclusions}

This review describes in a unique and wide perspective the complexity of the process of hysterectomy. We believe that systematic preoperative planning is of great importance for the outcome after hysterectomy and that individualized treatment may lead to improved postoperative outcomes. Despite great improvements in hysterectomy during recent decades, exemplified by the introduction of ERAS frameworks including less invasive surgical methods and stricter indications, the issue of preoperative planning has been largely unexplored. Prehabilitation measures could be developed and integrated with the ERAS concept, and together with assessment of preoperative goals, could form patient- and procedure specific pathways. Each part in the planning process should be further developed, and attention needs to be paid to promote research on the multimodality of the planning process. Randomized studies are warranted to evaluate the effect of the preoperative planning and preventive measures against adverse outcome in a systematic setting in order to improve the patient-experienced outcomes after hysterectomy for benign disease. The national societies in Obstetrics and Gynecology should be encouraged to promote the establishing of guidelines for the process of benign hysterectomy.

\section{Acknowledgements}

The study was supported financially by unrestricted grants from Region Östergötland and Linköping University. The funding sources were not involved in the study design, collection and analysis of data, report writing, or the decision to submit for publication.

\section{Conflict of Interest}

The authors declare that they have no conflict of interest.

\section{References}

[1] Hammer A, Rositch AF, Kahlert J et al. Global epidemiology of hysterectomy: possible impact on gynecological cancer rates. Am J Obstet Gynecol 2015; 213: 23-29

[2] Committee on Gynecologic Practice. Committee Opinion No. 701: Choosing the route of hysterectomy for benign disease. Obstet Gynecol 2017; 129: e155-e159

[3] Lefebvre G, Allaire C, Jeffrey J et al. SOGC clinical guidelines. Hysterectomy. Clinical practice gynaecology committee and executive committee and council, Society of obstetricians and gynaecologists of Canada. J Obstet Gynaecol Can 2002; 24: 37-61

[4] Moen M. Hysterectomy for benign conditions of the uterus: Total abdominal hysterectomy. Obstet Gynecol Clin North Am 2016; 43: 431440 
[5] Rannestad T, Eikeland O-J, Helland $\mathrm{H}$ et al. The quality of life in women suffering from gynecological disorders is improved by means of hysterectomy. Acta Obstet Gynecol Scand 2001; 80: 46-51

[6] Kho RM, Abrão MS. In Search for the best minimally invasive hysterectomy approach for the large uterus. Clin Obstet Gynecol 2017; 60: 286-295

[7] Persson P, Hellborg T, Brynhildsen J et al. Attitudes to mode of hysterectomy-a survey-based study among Swedish gynecologists. Acta Obstet Gynecol Scand 2009; 88: 267-274

[8] Kalogera E, Dowdy SC. Enhanced recovery pathway in gynecologic surgery: Improving outcomes through evidence-based medicine. Obstet Gynecol Clin North Am 2016; 43: 551-573

[9] Linkov F, Sanei-Moghaddam A, Edwards RP et al. Implementation of hysterectomy pathway: Impact on complications. Womens Health Issues 2017; 27: 493-498

[10] Pitter MC, Simmonds C, Seshadri-Kreaden U et al. The impact of different surgical modalities for hysterectomy on satisfaction and patient reported outcomes. Interact J Med Res 2014; 3: e11

[11] Persson P, Wijma K, Hammar M et al. Psychological wellbeing after laparoscopic and abdominal hysterectomy-a randomised controlled multicentre study. BJOG 2006; 113: 1023-1030

[12] Wodlin NB, Nilsson L, Kjølhede P. Health-related quality of life and postoperative recovery in fast-track hysterectomy. Acta Obstet Gynecol Scand 2011; 90: 362-368

[13] Bossick AS, Sangha R, Olden $\mathrm{H}$ et al. Identifying what matters to hysterectomy patients: Postsurgery perceptions, beliefs, and experiences. J Patient Cent Res Rev 2018; 5: 167-175

[14] Banugo P, Amoako D. Prehabilitaion. BJA Education 2017; 17: 401-405

[15] Schneider S, Armbrust R, Spies C et al. Prehabilitation programs and ERAS protocols in gynecological oncology: a comprehensive review. Arch Gynecol Obstet 2020; 301: 315-326

[16] Shamseer L, Moher D, Clarke M et al. Preferred reporting items for systematic review and meta-analysis protocols (PRISMA-P) 2015: Elaboration and explanation. BMJ 2015; 350: g7647

[17] Centre for Reviews and Dissemination (CRD). Systematic reviews; CRD's guidance for undertaking reviews in health care 2009 (internet). University of York: CRD; 2009. Accessed July 29, 2020 at: https://www.york.ac. uk/media/crd/Systematic_Reviews.pdf

[18] Kovac S, Barhan S, Lister M et al. Guidelines for the selection of the route of hysterectomy: Application in a resident clinic population. Am J Obstet Gynecol 2002; 187: 1521-1527

[19] Hullfish KL, Henry E, Ferguson JE. The effect of preoperative checklists on final histology and rates of hysterectomy for benign conditions. Female Pelvic Med Reconstr Surg 2012; 18: 143-147

[20] Radosa J, Radosa CG, Kastl C et al. Influence of the preoperative decision-making process on the postoperative outcome after hysterectomy for benign uterine pathologies. Geburtshilfe Frauenheilkd 2016; 76: 383-389

[21] Cheung LH, Callaghan P, Chang AM. A controlled trial of psycho-educational interventions in preparing Chinese women for elective hysterectomy. Int J Nurs Stud 2003; 40: 207-216

[22] Pakbaz M, Rolfsman E, Löfgren M. Are women adequately informed before gynaecological surgery? BMC Womens Health 2017; 17: 68
[23] Chapman GC, Nashar S, Billow M et al. Predictors of goal achievement in patients undergoing hysterectomy. Eur J Obstet Gynecol Reprod Biol 2019; 234: 1-5

[24] Perry F, Parker RK, White PF et al. Role of psychological factors in postoperative pain control and recovery with patient-controlled analgesia. Clin J Pain 1994; 10: 57-63

[25] Kain ZN, Sevarino F, Alexander GM et al. Preoperative anxiety and postoperative pain in women undergoing hysterectomy. J Psychosom Res 2000; 49: 417-422

[26] Ewalds-Kvist SBM, Hirvonen T, Kvist M et al. Depression, anxiety, hostility and hysterectomy. J Psychosom Obstet Gynecol 2005; 26: 193-204

[27] Hartmann KE, Ma C, Lamvu GM et al. Quality of life and sexual function after hysterectomy in women with preoperative pain and depression. Obstet Gynecol 2004; 104: 701-709

[28] Brandsborg B, Dueholm M, Nikolajsen L et al. A prospective study of risk factors for pain persisting 4 months after hysterectomy. Clin J Pain 2009; 25: $263-268$

[29] Pinto PR, Mcintyre T, Almeida A et al. The mediating role of pain catastrophizing in the relationship between presurgical anxiety and acute postsurgical pain after hysterectomy. Pain 2012; 153: 218-226

[30] Pokkinen SM, Nieminen K, Yli-Hankala A et al. Persistent posthysterectomy pain: A prospective, observational study. Eur ] Anaesthesiol 2015; 32: 718-724

[31] Theunissen M, Peters ML, Schepers J et al. Recovery 3 and 12 months after hysterectomy: epidemiology and predictors of chronic pain, physical functioning, and global surgical recovery. Medicine (Baltimore) 2016; 95: e3980

[32] As-Sanie S, Till SR, Mowers EL et al. Opioid prescribing patterns, patient use, and postoperative pain after hysterectomy for benign indications. Obstet Gynecol 2017; 130: 1261-1268

[33] Weber AM, Walters MD, Schover LR et al. Functional outcomes and satisfaction after abdominal hysterectomy. Am J Obstet Gynecol 1999; 181: 530-535

[34] Kuppermann M, Learman LA, Schembri M et al. Predictors of hysterectomy use and satisfaction. Obstet Gynecol 2010; 115: 543-551

[35] Rahkola-Soisalo P, Brummer T, Jalkanen J et al. Hysterectomy provides benefit in health-related quality of life: a 10-year follow-up study. Minim Invasive Gynecol 2020; 27: 868-874

[36] Hughes M, Hackney R, Lamb P et al. Prehabilitation before major abdominal surgery: A systematic review and meta-analysis. World J Surg 2019; 43: $1661-1668$

[37] Ebner F, Schulz SVW, de Gregorio A et al. Prehabilitation in gynecologica surgery? What do gynecologists know and need to know. Arch Gynecol Obstet 2018; 297: 27-31

[38] Thomsen T, Villebro N, Møller AM. Interventions for preoperative smok ing cessation. Cochrane Database Syst Rev 2014; (3): CD002294

[39] Oppedal K, Møller AM, Pedersen B et al. Preoperative alcohol cessation prior to elective surgery. Cochrane Database Syst Rev 2012; (7): CD008343

[40] Hulzebos EHJ, Smit Y, Helders PPJM et al. Preoperative physical therapy for elective cardiac surgery patients. Cochrane Database Syst Rev 2012; (11): CD010118

[41] Bohlin KS, Ankardal M, Stjerndahl J-H et al. Influence of the modifiable life-style factors body mass index and smoking on the outcome of hysterectomy. Acta Obstet Gynecol Scand 2016; 95: 65-73 